\title{
Recreating the seawater mixture composition of HOCs in toxicity tests with Artemia franciscana by passive dosing
}

\author{
E. Rojo-Nieto ${ }^{a}{ }^{*}$, K.E.C. Smith ${ }^{\text {b }}$, J.A. Perales ${ }^{a}$, P. Mayer $^{b}$ \\ a Andalusian Centre of Marine Science and Technology (CACYTMAR), Department of Environmental Technologies, University of Cadiz, 11510 Puerto Real, Spain \\ ${ }^{\mathrm{b}}$ Department of Environmental Science, Aarhus University, DK-4000 Roskilde, Denmark
}

\section{A R T I C L E I N F O}

\section{Article history:}

Received 21 February 2012

Received in revised form 9 April 2012

Accepted 11 April 2012

\section{Keywords:}

Passive dosing

PAH toxicity

Artemia franciscana

Seawater

Mixtures

Chemical activity

\begin{abstract}
A B S T R A C T
The toxicity testing of hydrophobic organic compounds (HOCs) in aquatic media is generally challenging, and this is even more problematic for mixtures. The hydrophobic properties of these compounds make them difficult to dissolve, and subsequently to maintain constant exposure concentrations. Evaporative and sorptive losses are highly compound-specific, which can alter not only total concentrations, but also the proportions between the compounds in the mixture. Therefore, the general aim of this study was to explore the potential of passive dosing for testing the toxicity of a PAH mixture that recreates the mixture composition found in seawater from a coastal area of Spain, the Bay of Algeciras. First, solvent spiking and passive dosing were compared for their suitability to determine the acute toxicity to Artemia franciscana nauplii of several PAHs at their respective solubility limits. Second, passive dosing was applied to recreate the seawater mixture composition of PAHs measured in a Spanish monitoring program, to test the toxicity of this mixture at different levels. HPLC analysis was used to confirm the reproducibility of the dissolved exposure concentrations for the individual PAHs and mixtures. This study shows that passive dosing has some important benefits in comparison with solvent spiking for testing HOCs in aquatic media. These include maintaining constant exposure concentrations, leading to higher reproducibility and a relative increase in toxicity. Passive dosing is also able to faithfully reproduce real mixtures of HOCs such as PAHs, in toxicity tests, reproducing both the levels and proportions of the different compounds. This provides a useful approach for studying the toxicity of environmental mixtures of HOCs, both with a view to investigating their toxicity but also for determining safety factors before such mixtures result in detrimental effects.
\end{abstract}

(ㄷ) 2012 Elsevier B.V. All rights reserved.

\section{Introduction}

Due to their properties and the environmental implications, 7 polycyclic aromatic hydrocarbons (PAHs) are included in the list of Priority Pollutants of the European Water Framework Directive (Council Directive 2008/105/EC, 2008) and 16 PAHs in the United States Environmental Protection Agency (USEPA) Priority Pollutant List (Code of Federal Regulation, 1982). PAHs are characterized by low solubilities in aqueous media, and once dissolved in water they tend to partition to organic phases including living organisms (de Maagd et al., 1998). The levels of PAHs in surface seawater can vary widely. For example, Witt (2002) found concentrations of up to $0.0166 \mu \mathrm{g} \mathrm{L}^{-1}$ in the Central Baltic Sea ( $\Sigma 15$ PAHs). In the Bahia Blanca estuary (Argentina), levels for $\Sigma 17$ PAHs ranged from "undetected" to more than $4 \mu \mathrm{gL}^{-1}$ (Arias et al., 2009). In a study

\footnotetext{
* Corresponding author. Tel.: +34 956016587; fax: +34 956016746

E-mail address: elisa.rojo@uca.es (E. Rojo-Nieto).
}

of seawater around England and Wales, concentrations as high as $10.7 \mu \mathrm{g} \mathrm{L}^{-1}$ ( $\Sigma 15 \mathrm{PAHs}$ ) were found in unfiltered water (Law et al., 1997). It is not only the heterogeneity in concentrations within a region that results in different exposure, but also the composition of the PAH mixture profile varies. This is rarely addressed in traditional risk assessment and management, which focus on the exposure and effects of single compounds and which might lead to an underestimation of the actual risks (Altenburger and Greco, 2009). It is therefore essential to develop and apply new methods to study the exposure and toxicity of complex mixtures that resemble real cases. Moreover, in field samples it is often of interest to investigate the toxicity of a specific group of chemicals such as PAHs. However, such field samples also contain other pollutants in addition to the target pollutants, which make it practically impossible to determine their toxicity directly using field samples.

The toxicity testing of hydrophobic organic compounds (HOCs) in aquatic media is generally challenging, because the hydrophobic properties of these compounds makes them difficult to dissolve and to maintain constant exposure throughout the test (ECETOC, 
1996 and OECD, 2000). An additional challenge is to test these substances at the solubility level, while avoiding crystals or microdroplets of the test substance in the test medium. The conventional dosing technique is spiking at the beginning of the test using a cosolvent, but here the concentrations might still decrease during the test and furthermore the solvent might contribute to or modify the toxicity of the test compounds (Hutchinson et al., 2006). This difficulty in maintaining constant concentrations is even more problematic for mixtures, because compound-specific losses can alter the mixture composition during the test. Passive dosing has recently been introduced for establishing and controlling exposure levels by equilibrium partitioning from a dominating donor phase (Bandow et al., 2009; Brown et al., 2001; Kiparissis et al., 2003; Mayer et al., 1999; Smith et al., 2010a). A key feature of passive dosing is that it allows freely dissolved concentrations and chemical activities to be controlled throughout the test. It has recently been proposed, experimentally confirmed, that chemical activity is a key parameter for the baseline toxicity of hydrophobic organic chemicals, and that some highly hydrophobic substances can exert baseline toxicity at very low aqueous concentrations but at relatively high chemical activities (Engraff et al., 2011; Mackay et al., 2009, 2011; Mayer and Holmstrup, 2008; Mayer and Reichenberg, 2006; Reichenberg and Mayer, 2006; Smith et al., 2010a). Diffusion across membranes occurs spontaneously down chemical activity rather than concentration gradients, with equilibrium partitioning being defined by equal chemical activities (Mayer and Reichenberg, 2006). Chemical activity is not related to total concentrations, but with the freely dissolved concentration $\left(C_{\text {free }}\right)$, which can be understood as the concentration of freely dissolved molecules (not sorbed or bound to other phases).

The first aim of this study was to compare co-solvent spiking and passive dosing in a marine toxicity test with Artemia franciscana Kellogg (1906) (brine shrimp). These experiments were done in parallel and under identical conditions for the optimal comparison of the two dosing techniques. The second aim was to determine the toxicity of 10 PAHs to brine shrimp at exactly their respective solubility limits, and to compare the results to other tested organisms. The third aim was to explore the potential of passive dosing for recreating the composition of an environmental HOC mixture in an aquatic toxicity test.

\section{Materials and methods}

\subsection{Chemicals and materials}

Naphthalene (99\%, Aldrich, Germany), acenaphthene (99\%, Fluka, Germany), fluorene (99\%, Fluka), phenanthrene (>99.5\%, Aldrich), anthracene (99\%, Fluka), pyrene (>99\%, Sigma, Germany), fluoranthene (99\%, Aldrich), benz[a]anthracene (99\%, Aldrich), benzo(a)pyrene (98\%, Cerilliant, TX, USA) and chrysene (99\%, Cerilliant) were used. Ten milliliter autosampler vials with Teflon lined screw caps were bought from Mikrolab (Aarhus, Denmark). Medical grade PDMS silicone was made using the MDX4-4210 kit from Dow Corning, supplied by the Institute of Anaplastology (Velten, Germany). Lint-free lens tissue was used for cleaning (Bie and Bernsten A/S, Denmark). Methanol (HPLC grade) was used for extraction and analysis (Merck, Darmstatd, Germany) and MilliQ water (Super Q treated, Millipore, MA, USA) for rinsing.

\subsection{Artificial seawater}

Artificial seawater was prepared by dissolving $\mathrm{NaCl}$ (21.03 $\left.\mathrm{g} \mathrm{L}^{-1}\right), \mathrm{Na}_{2} \mathrm{SO}_{4}\left(3.52 \mathrm{~g} \mathrm{~L}^{-1}\right), \mathrm{KCl}\left(0.61 \mathrm{~g} \mathrm{~L}^{-1}\right), \mathrm{KBr}\left(0.088 \mathrm{~g} \mathrm{~L}^{-1}\right)$, $\mathrm{Na}_{2} \mathrm{~B}_{4} \mathrm{O}_{7} \times 10 \mathrm{H}_{2} \mathrm{O}\left(0.034 \mathrm{~g} \mathrm{~L}^{-1}\right), \quad \mathrm{MgCl}_{2} \times 6 \mathrm{H}_{2} \mathrm{O}\left(9.5 \mathrm{gL}^{-1}\right)$, $\mathrm{CaCl}_{2} \times 2 \mathrm{H}_{2} \mathrm{O}\left(1.32 \mathrm{~g} \mathrm{~L}^{-1}\right), \mathrm{SrCl}_{2} \times 6 \mathrm{H}_{2} \mathrm{O}\left(0.02 \mathrm{~g} \mathrm{~L}^{-1}\right)$, and $\mathrm{NaHCO}_{3}$ $\left(0.17 \mathrm{~g} \mathrm{~L}^{-1}\right)$ in Millipore water as recommended by the USEPA for acute toxicity tests with marine organisms (USEPA, 2002). The final total salt concentration was $0.45 \mathrm{M}$.

\subsection{Artemia franciscana nauplii individuals for toxicity tests}

Toxicity assays were carried out using a homogeneous $A$. franciscana population that had been incubated in the same medium and grown synchronously. Certified cysts of $A$. franciscana nauplii (AF450, INVE) were bought from Acuazul, S.C. (Spain). Cysts were hatched at $20^{\circ} \mathrm{C}$ in artificial seawater and under artificial light illumination with a light/dark period of $16 / 8 \mathrm{~h}$. Hatching was carried out under conditions that were as similar as possible to conditions in the toxicity tests. The only parameter that was changed between the hatching procedure and toxicity assays was light, since according to the Food and Agriculture Organization of the United Nations (FAO) (Sorgeloos et al., 1986) illumination is essential for optimal hatching of Artemia cysts, at least for the first hours after hydration. Newborn nauplii were isolated under a stereoscopic microscope with a Pasteur pipette, and 10 nauplii of less than 5-h-old added to each passive dosing or spiking vial.

As has been suggested by several authors (Hadjispyrou et al., 2001; Koutsaftis and Aoyama, 2007; Nunes et al., 2006; Sarabia et al., 2002) there are a number of reasons for the selection of brine shrimp, Artemia sp., as a test organism. It is gaining popularity as a test organism because of the continuous availability of Artemia in the form of dormant eggs (cyst), and its adaptability to wide ranges of salinity and temperature. Since test animals hatching from cysts are of a similar age, genotype and physiological condition the test variability is greatly reduced, guaranteeing reliability, feasibility and cost effectiveness in routine and/or research ecotoxicity tests. For all these reasons, Artemia, or more precisely A. francisacana, has been chosen as test organism for the acute assays in artificial sea water in this study. The chosen end point was mortality of the recently (instar II-III stage) hatched nauplii, a well-accepted end point in cyst-based toxicity assays with Artemia (Nunes et al., 2006).

\subsection{Preparation and loading of the passive dosing vials}

The PDMS silicone pre-polymer and catalyst were mixed according to the supplier, and $500 \mathrm{mg}( \pm 1 \%)$ were cast into the base of the $10 \mathrm{~mL}$ autosampler vials. These were then stored overnight at $4{ }^{\circ} \mathrm{C}$ to allow any air bubbles formed during casting to escape, before placing in an oven at $110^{\circ} \mathrm{C}$ for $48 \mathrm{~h}$ to cure. The cured silicone was rinsed 3 times with methanol to remove oligomers and other impurities, followed by 3 rinses with MilliQ water to remove any adhering methanol. Finally, the surfaces were dried with lint-free tissue.

For the single PAH toxicity assays and salting-out experiments (conducted to know the exact solubility of PAHs in this specific artificial seawater), passive dosing vials were loaded to saturation with individual PAHs using methanol suspensions of each compound (Smith et al., 2010a). The PAH crystals in the methanol suspension served to maintain the saturation level in the methanol and thus also in the silicone. For the PAH mixture toxicity assays, the PDMS silicone in the vials was loaded to below saturation using mixtures of the PAHS dissolved in methanol at the appropriate concentrations. The volume of methanol PAH mixture solution used was sufficient to ensure negligible depletion during the loading process. The PAH mixture profile was taken from an unfiltered superficial sea water sample, collected as part of a monitoring program from a coastal area of Spain, in the Bay of Algeciras.

Measured values of the $\mathrm{PAH}$ equilibrium partitioning ratios between PDMS silicone and the seawater used in this study, 
$K_{\mathrm{PDMS}}$ Seawater $\left(\mathrm{LL}^{-1}\right)$, were not available. These were therefore calculated using Eq. (1):

$K_{\text {PDMS:Seawater }}=\frac{C_{\text {PDMS,sat }}}{C_{\text {Seawater,sat }}}$

PAH saturation concentrations in seawater, $C_{\text {Seawater,sat }}\left(\mathrm{mg} \mathrm{L}^{-1}\right)$, were measured in the salting-out experiments performed using PAH saturated passive dosing vials (see details below). The corresponding saturation silicone concentrations, $C_{\mathrm{PDMS} \text {,sat }}\left(\mathrm{mg} \mathrm{L}^{-1}\right)$ were not measured and were calculated using Eq. (2):

$C_{\mathrm{PDMS}, \mathrm{sat}}=\frac{C_{\mathrm{MeOH}, \mathrm{sat}}}{K_{\mathrm{MeOH}: \mathrm{PDMS}}}$

where $C_{\mathrm{MeOH} \text {,sat }}\left(\mathrm{mg} \mathrm{L}^{-1}\right.$, from Reichenberg et al., 2008) is the PAH solubility in MeOH and $K_{\mathrm{MeOH}}$ PDMS ( $\mathrm{LL}^{-1}$, from Smith et al., 2010a) is the PAH equilibrium partitioning ratio between methanol and PDMS (Mackay et al., 2006; Table S1).

For passive dosing to reproduce PAH concentrations of field samples in the laboratory requires that the PDMS silicone is loaded to the correct concentration, $C_{\mathrm{PDMS}}\left(\mathrm{mg} \mathrm{L}^{-1}\right)$.

$C_{\text {PDMS }}=C_{\text {Seawater }} \times K_{\text {PDMS:Seawater }}$

where $C_{\text {Seawater }}\left(\mathrm{mg} \mathrm{L}^{-1}\right)$ is the seawater PAH concentration from the monitoring sample and $K_{\text {PDMS:Seawater }}$ is from above.

Finally, loading the PDMS silicone to the correct value of $C_{\mathrm{PDMS}}$ requires that the methanol loading solution has the correct concentration, $C_{\mathrm{MeOH}}\left(\mathrm{mg} \mathrm{L}^{-1}\right)$. This was calculated using Eq. (4) below:

$C_{\mathrm{MeOH}}=\frac{C_{\mathrm{PDMS}}}{K_{\mathrm{PDMS}: \mathrm{MeOH}}}$

\subsection{Tests and analysis}

\subsubsection{Salting out}

Solubilities of PAHs at the specific temperature and salinity of the artificial seawater were necessary to prepare and carry out all tests. To measure the salting out effect, and calculate the Setschenow constants, for the 10 PAHs at $20^{\circ} \mathrm{C}$, the aqueous solubilities in MilliQ water and artificial seawater were measured $(n=5)$. Passive dosing vials were loaded to saturation with each PAH as described above, filled with $5 \mathrm{~mL}$ of MilliQ water and vortexed for $1 \mathrm{~h}(1000 \mathrm{rpm})$ in the dark at $20^{\circ} \mathrm{C}$. This was sufficient for equilibrium to be reached between the silicone and water (Smith et al., 2010a). A $500 \mu \mathrm{L}$ sample of water from each vial was mixed with $500 \mu \mathrm{L}$ of methanol, and analyzed by HPLC. The remaining MilliQ water was poured off, and the vial surfaces cleaned using lint free tissue and rinsed 3 times with artificial sea water. Five milliliter of artificial seawater were then added, and the same equilibration, sampling and analysis procedure as described for MilliQ water followed.

\subsubsection{PAH toxicity to A. franciscana}

Loaded passive dosing vials were each filled with $5 \mathrm{~mL}$ of artificial seawater and vigorously shaken for $1 \mathrm{~h}$ (1000 rpm) in the dark at $20^{\circ} \mathrm{C}$, before leaving for a further $24 \mathrm{~h}$ to ensure equilibrium was reached. For the spiking experiments, $10 \mathrm{~mL}$ autosampler vials were filled with $5 \mathrm{~mL}$ of artificial seawater and spiked with PAH solutions in methanol to the saturation concentration in seawater. Previously, tests with methanol were carried out to determine the concentrations of methanol having no effect to A. franciscana, and these showed that methanol did not exert significant mortality when below 2.5\% (v/v). The concentrations of PAHs in the methanol spiking solutions therefore needed to be high enough to ensure that the volume of methanol added was below this amount, with the maximum amount added being $1 \%(\mathrm{v} / \mathrm{v})$. This meant that 3 PAHs (chrysene, benzo(a)pyrene and benzo(a)anthracene) could not be properly tested by co-solvent spiking, since their low solubilities in water resulted in crystal precipitation when the highly concentrated methanol solutions required to reach saturation in water were added. To avoid this would have required using less concentrated methanol solutions, but in this case the volumes of methanol needed were too high. Two types of control were included in all experiments. One control treatment $(n=10)$ consisted of $10 \mathrm{~mL}$ autosampler vials with no PDMS in the base and spiked with $1 \%$ $(\mathrm{v} / \mathrm{v})$ clean methanol. A second control $(n=10)$ was conducted in passive dosing vials with PDMS, which were loaded with clean methanol. Each treatment (individual PAHs and their mixtures in both the passive dosing and spiked experiments) was tested in quintuplicate.

\subsubsection{Exposure confirmation}

At the end of the passive dosing tests using individual PAHs and their mixtures, exposure was confirmed by equilibrating the testing vials with $1 \mathrm{~mL}$ of MilliQ water and measuring the final freely dissolved concentrations. For the assays with individual PAHs at saturation, equilibrating with pure water rather than the artificial seawater means that these values can be directly compared to literature aqueous solubilities as a cross-check that the saturation was in fact obtained during loading and that there was no significant depletion during the test. These aqueous solubilities in pure water were then converted into seawater concentrations using the above obtained Setschenow constants for the discussion. PAH analyses were carried out according to Smith et al. (2010a), by HPLC with fluorescence detection (Agilent 1100 HPLC equipped with a G1321A FLD).

\subsubsection{Data analysis}

The chemical activity ( $a$ ) of PAHs in saturated water is equal to the activity of the PAHs in their pure solid crystal state. In the tests with single PAHs, passive dosing was applied to control the freely dissolved concentration at aqueous solubility, this being equivalent to the testing at maximum chemical activity $\left(a_{\max }\right)$. The crystal activities were estimated from the compound's melting point $\left(T_{\mathrm{m}}\right)$ assuming the entropy of melting to be $56 \mathrm{~J} \mathrm{~mol}^{-1} \mathrm{~K}^{-1}$, i.e. Walden's rule (Smith et al., 2010a; Mayer and Holmstrup, 2008).

$a_{\max }=\exp \left(6.8\left[1-\frac{T_{\mathrm{m}}}{T}\right]\right)$

where $a_{\max }$ is the maximum chemical activity (unitless), $T_{\mathrm{m}}$ is the temperature of melting point $(\mathrm{K})$ and $T$ is the temperature of the tests $(293 \mathrm{~K})$.

The observed mortality of $A$. franciscana nauplii was plotted against $a_{\max }$ of the PAHs, using GraphPad Prism 5 (San Diego, CA). A sigmoidal dose-response curve was fitted to the mortalities of singe PAHs.

To translate the dissolved exposure concentrations of the individual PAHs in the seawater mixture experiments into chemical activities requires that these concentrations be divided by their respective subcooled liquid solubilities. However, literature subcooled liquid solubilities apply to pure water only. Therefore the data from the salting out experiment were used to calculate PAH subcooled liquid solubilities for the artificial sea water, $S_{\mathrm{L}, \text { Seawater }}$ ( $\mathrm{mg} \mathrm{L}^{-1}$ ), using Eq. (6):

$S_{\mathrm{L}, \text { Seawater }}=\frac{C_{\text {Seawater,sat }}}{a_{\max }}$

where $C_{\text {Seawater,sat }}\left(\mathrm{mg} \mathrm{L}^{-1}\right)$ is the measured solubility of each PAH in seawater from the salting out experiment, and values of $a_{\max }$ for each PAH were calculated using Eq. (4). These values of $S_{L, \text { seawater }}$ were then used to convert the passive dosing reproduced dissolved 
concentrations, $C_{\text {free(Seawater), }}$ into their respective chemical activities, a (unitless), using Eq. (7):

$a=\frac{C_{\text {free(Seawater) }}}{S_{\mathrm{L}, \text { Seawater }}}$

\section{Results and discussion}

\subsection{Individual PAHs at saturation}

\subsubsection{Passive dosing}

The toxicity tests were carried out in artificial sea water, at $20^{\circ} \mathrm{C}$. With passive dosing, the PAH chemical activities depend on the loading and testing temperatures (Eq. (5)). In this study, these were the same and therefore chemical activities for the different PAHs were the same in MilliQ and artificial sea water. However, despite these same chemical activities, PAH concentrations are different in both media due to the salting out effect. To translate the measured concentrations in MilliQ water to sea water concentrations, passive dosing was used to quantify the salting out effect for the artificial sea water. Table 1 shows equilibrium concentrations in MilliQ water and in artificial seawater for the different PAHs, together with the corresponding Setschenow constants calculated using the Setschenow equation (Setschenow, 1889):

$\log \frac{S}{S_{0}}=-K_{\text {salt }} \times C_{\text {salt }}$

where $S$ and $S_{0}$ are the solubilities of the organic solute in aqueous salt solution and in water, respectively, $C_{\text {salt }}$ is the molar concentration of electrolyte and $K_{\text {salt }}$ is the empirical Setschenow constant.

The latter give the PAH-specific salting-out effect in the artificial sea water, with these values being similar to those obtained by Jonker and Muijs (2010) and Gouliarmou et al. (2012), who studied this effect in different artificial sea waters. Differences between the concentrations in MilliQ water and in artificial sea water are shown in Table 1, and all PAHs showed a decreased solubility in artificial sea water compared to MilliQ water by a factor of between 0.22 and 0.29 .

Exposure confirmation of the final freely dissolved concentration from the passive dosing experiments was carried out by equilibrating with MilliQ water, and compared with literature data at $20^{\circ} \mathrm{C}$. In Fig. S1, the final PAH equilibrium water concentrations at the end of the test are plotted against their literature aqueous solubilities and lie close to the 1:1 line. They also have a high reproducibility, as is shown in Table 1 by the coefficients of variation (CV). This indicates that the loading procedure did indeed saturate the PDMS to give equilibrium water concentrations close to aqueous solubility, and furthermore that the buffering capacity of the PDMS was sufficient during the test. The passive dosing approach is therefore useful for aquatic toxicity testing exactly at aqueous solubility (limit testing) for screening purpose, which may eventually be useful in reducing the number of tests. A further advantage is the portability of the passive dosing approach, allowing toxicity experiments to be carried out on site at different locations. In this study, some of the passive dosing experiments were carried out in Denmark and the remainder in Spain.

\subsubsection{Solvent spiking versus passive dosing in toxicity tests}

For studying the aquatic toxicity of hydrophobic organic pollutants, one of most commonly used techniques is spiking using solvent. Passive dosing and spiking were therefore compared in parallel toxicity assays, to determine the advantages and limitations of each approach for aquatic toxicity tests. The toxicity to A. franciscana nauplii of 7 individual PAHs at aqueous solubilities after $72 \mathrm{~h}$ dark exposure in artificial sea water at $20^{\circ} \mathrm{C}$ was compared for passive dosing and solvent spiking (Fig. 1). The measured

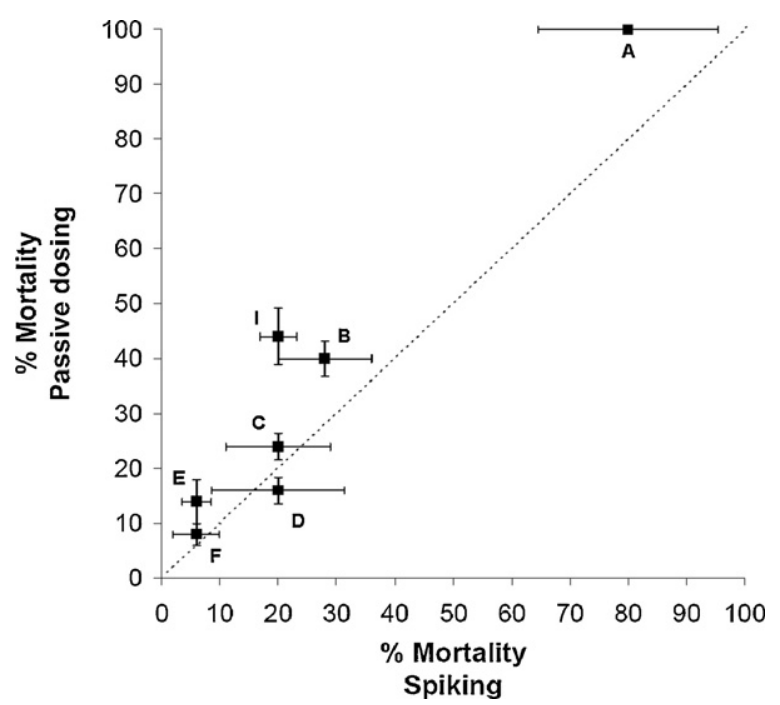

Fig. 1. Mortality of $A$. franciscana nauplii and reproducibility obtained by passive dosing and spiking solvent, at $20^{\circ} \mathrm{C}$ and $0.45 \mathrm{M}$ salinity. Individual PAHs ordered by decreasing maximum chemical activity were: (A) naphthalene, (B) acenaphthene, (C) phenanthrene, (D) fluoranthene, (E) fluorene, (F) pyrene and (I) anthracene. Three PAHs (benzo(a)pyrene, benzo(a)anthracene and chrysene) could not tested by spiking and were only tested by passive dosing. The error bars indicate the standard error of the mean (SEM) from the quintuplicates.

toxicities showed the same general trend, but particularly for the lighter PAHs a higher toxicity was observed with passive dosing. This is because exposure concentrations were maintained by repartitioning from the PDMS, which was not the case with spiking. With spiking there is therefore the risk that the toxicity of such compounds might be underestimated. With solvent spiking there was also a slightly higher variation between replicate samples. Spiking has the added disadvantage of including solvent in the water, which could potentially change the test compound solubility and also interfere with the toxicity. In fact, testing at aqueous solubility could not be carried out for three PAHs using solvent spiking. Their limited solubilities in water, in conjunction with the large difference to their methanol solubilities, resulted in crystal precipitation in the seawater when methanol with the required high concentrations of PAHs was used. This could not be circumvented by adding more dilute methanol solutions, since the volume of methanol was then high enough to result in solvent toxicity. For these compounds passive dosing offers a suitable approach for testing at aqueous solubility. Therefore, the passive dosing approach offers some advantages for toxicity testing: freely dissolved exposure levels are maintained throughout the test period, leading to a higher reproducibility and for some compounds a higher measured toxicity.

\subsubsection{Effects of single compounds in A. franciscana nauplii}

Hydrophobic organic compounds often exert their effects by what is known as baseline toxicity. The impairment of membrane function occurs when the compounds reach a critical membrane concentration (van Wezel and Opperhuizen, 1995). The partitioning of organic compounds into the cellular phospholipid membranes of $A$. franciscana nauplii is a spontaneous process driven by the partial molar free energy, which can be quantified by its chemical activity. Therefore, the toxicity data from the passive dosing study were used to investigate the relationship between toxicity and chemical activity. The controls all showed $100 \%$ survival (data not shown). Fig. 2 shows the percentage mortality of $A$. franciscana nauplii plotted against the PAH maximum chemical activities. The mortality data for all individual PAHs could be fitted to one single chemical activity-response curve $\left(R^{2}=0.94\right)$ given by the equation 
Table 1

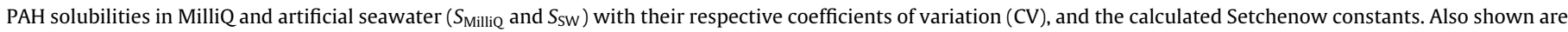
the crystal activities $\left(a_{\text {crystal }}\right)$ and subcooled liquid solubilities $\left(S_{\mathrm{L}}\right)$ at the test conditions $\left(T=20^{\circ} \mathrm{C}\right.$ and salinity $\left.=0.45 \mathrm{M}\right)$.

\begin{tabular}{|c|c|c|c|c|c|c|c|}
\hline $\mathrm{PAH}$ & $S_{\text {MilliQ }}\left(\mu \mathrm{g} \mathrm{L}^{-1}\right)$ & $\mathrm{CV}_{\text {MilliQ }}$ & $S_{\mathrm{SW}}\left(\mu \mathrm{g} \mathrm{L}^{-1}\right)$ & $\mathrm{CV}_{\text {Sea water }}$ & Setschenow constant & $a_{\text {crystal }}$ & $S_{\mathrm{L} \text { Sea water }}\left(\mu \mathrm{g} \mathrm{L}^{-1}\right)$ \\
\hline Naphthalene & $32,201.41$ & 0.06 & $25,244.31$ & 0.07 & 0.23 & 0.244 & $98,929.754$ \\
\hline Acenaphthene & 2434.00 & 0.01 & 1862.53 & 0.02 & 0.26 & 0.180 & $13,711.696$ \\
\hline Fluorene & 1480.91 & 0.03 & 1111.62 & 0.05 & 0.28 & 0.119 & $12,421.414$ \\
\hline Pyrene & 92.07 & 0.05 & 65.76 & 0.01 & 0.32 & 0.047 & 1803.861 \\
\hline Phenanthrene & 629.74 & 0.01 & 451.02 & 0.02 & 0.32 & 0.161 & 3753.177 \\
\hline Fluoranthene & 157.88 & 0.02 & 115.22 & 0.01 & 0.30 & 0.125 & 1244.560 \\
\hline Anthracene & 35.98 & 0.02 & 26.11 & 0.02 & 0.31 & 0.011 & 3258.158 \\
\hline Benzo(a)anthracene & 6.34 & 0.07 & 4.55 & 0.02 & 0.32 & 0.039 & 272.398 \\
\hline Benzo(a)pyrene & 0.96 & - & 0.69 & 0.05 & 0.32 & 0.029 & 23.989 \\
\hline
\end{tabular}

$Y=100 /\left(1+10^{(\operatorname{logEC} 50-X)} \times\right.$ hill slope $)$, except for anthracene that showed some excess toxicity.

Interestingly, the same enhanced toxicity for anthracene was observed in the spiking experiments (Fig. 1), suggesting that this is a real phenomenon. This could be due to the presence of metabolites of this compound, which, in the case of anthracene, have been found to dramatically increase toxicity in comparison to parent chemical alone (Mallakin et al., 1999). These metabolites could have been generated in two different ways: photooxidation or metabolism. According to Huang et al. (1997) anthracene photooxidation products are similar to those produced by biological oxidation via cytochrome P450. Experiments were conducted in darkness, but a brief exposure to laboratory light could have occurred during the introduction of the organisms into the vials. However, phototoxicity can be ruled out, because photoinduced anthracene toxicity does not occur under fluorescent laboratory lighting (Allred and Giesy, 1985). Ruling out photooxidation, the other possible way of metabolite formation is by Artemia sp. metabolism. According to Rewitz et al. (2006) the P450 enzyme systems (which mediate Phase I metabolism of PAHs) play a pivotal role in the physiology of in particular marine invertebrates compared to other organisms, catalyzing not only the metabolism of xenobiotics but also the biosynthesis of signal molecules, including the molting hormone in crustaceans (such as Artemia sp.). The A. franciscana nauplii pass several larval stages and several molts between the beginning ( $5 \mathrm{~h}-$ old) and the end ( $72 \mathrm{~h}$ later) of the assays (Lavens and Sorgeloos, 1996; Warner and Matheson, 1998). This involves the generation of molting hormones, and an increased activity of the P450 enzymes.

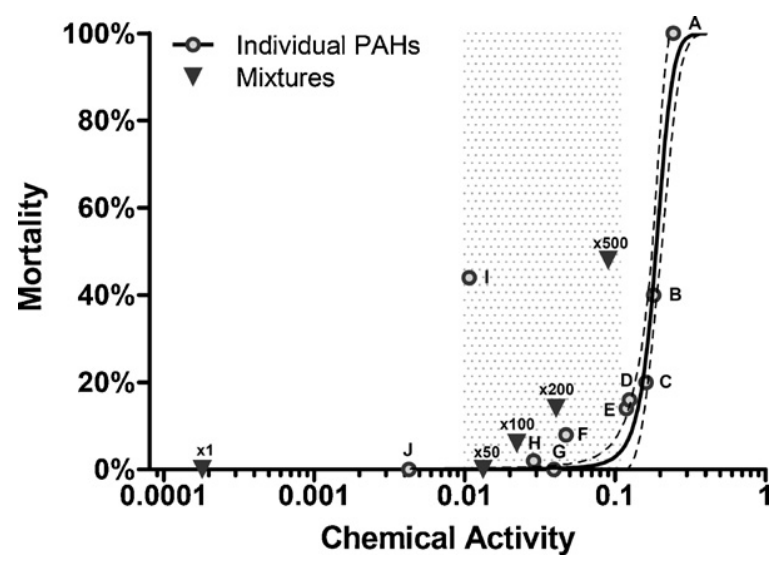

Fig. 2. Mortality of A.franciscana nauplii when exposed to different PAHs and mixtures, at $20^{\circ} \mathrm{C}$ and $0.45 \mathrm{M}$ salinity. Individual PAHs ordered by decreasing maximum activity were: (A) naphthalene, (B) acenaphthene, (C) phenanthrene, (D) fluoranthene, (E) fluorene, $(F)$ pyrene, $(G)$ benzo(a)anthracene, $(H)$ benzo(a)pyrene, (I) anthracene and $(\mathrm{J})$ chrysene, and were tested at saturation concentration $\left(a_{\max }\right)$. Mixtures were multiples of monitoring seawater concentrations. The grey area illustrates effective range for baseline toxicity observed in other studies. The sigmoidal curve is based on the mortality data of individual PAHs, with the dotted lines giving the $95 \%$ confidence interval of the curve.
This might also lead to an increased metabolism of compounds (such as the PAHs) and thus the generation of their metabolites, this being higher than normally found outside of periods of metamorphosis. In fact, Stevenson (2003) studied PAH metabolism in different marine organisms, and showed that crustacean Phase I metabolism of PAHs was relatively high. On the other hand, Di Toro and McGrath (2000) proposed that metabolites of narcotic chemicals, such as the PAHs, often exert a toxic mode of action similar to the parent compounds (i.e. non-polar narcosis), and that a large portion of metabolites often will remain in the body. This theory was applied successfully to a body-burden model based on equilibrium partitioning, supporting it (Mathew et al., 2008). The presence of some PAH metabolism does not necessarily contradict the findings of this study, even when taking into account the hypothesis presented by Di Toro and McGrath. Major deviations would only be observed for those PAHs whose metabolites show a much higher acute toxicity than the parent compound (such as anthracene), and only for organisms with a high PAH metabolism potential, such as $A$. franciscana in first stages of development. The higher than expected toxicity of anthracene based on its low maximum chemical activity has been observed in another passive dosing study with marine crustaceans in seawater (Engraff et al., 2011), but not in similar terrestrial and freshwater tests (Mayer and Holmstrup, 2008; Smith et al., 2010a).

The highlighted zone in Fig. 2 shows the $0.01-0.1$ range in effective chemical activities causing $50 \%$ mortality (Ea-50s) found in other studies using different media and organisms (Mackay et al., 2009; Mayer and Holmstrup, 2008; Mayer and Reichenberg, 2006; Smith et al., 2010a, 2010b). In this study the Ea-50 determined from fitting of the individual PAH mortality data (both cases, anthracene included as excluded) was 0.187 (equal in both, with a 95\% CI of $0.174-0.199$ and $0.181-0.193$ respectively). This is higher than the above mentioned range previously observed in the literature and might be explained in different ways. A. franciscana is a highly resistant organism and therefore might have a lower susceptibility to PAH narcotic toxicity than other organisms. Additionally the $72 \mathrm{~h}$ exposure period might not have been sufficient for equilibrium to be reached between the organisms' membranes and surrounding aqueous environment. Mortality is of course a very drastic endpoint, and it would have been interesting to be able to quantify toxicity using other more sensitive endpoints. In fact, other sublethal toxic effects such as convulsions or erratic swimming were observed at chemical activities between 0.01 and 0.1 , but unfortunately it was not possible to quantify these in a reliable manner.

\subsection{PAH mixtures}

\subsubsection{Reproduction of real seawater}

The third aim of this study was using passive dosing for the proper reproduction of a real seawater mixture of seven PAHs for toxicity testing purposes. This was focused both on reproducing the actual proportions of each compound found in the real 
Table 2

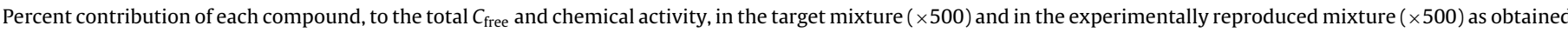

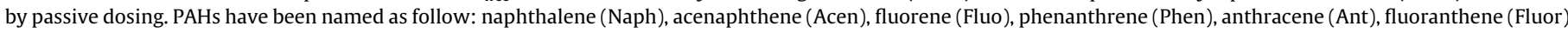
and pyrene (Pyr). *Indicates compound below detection limits.

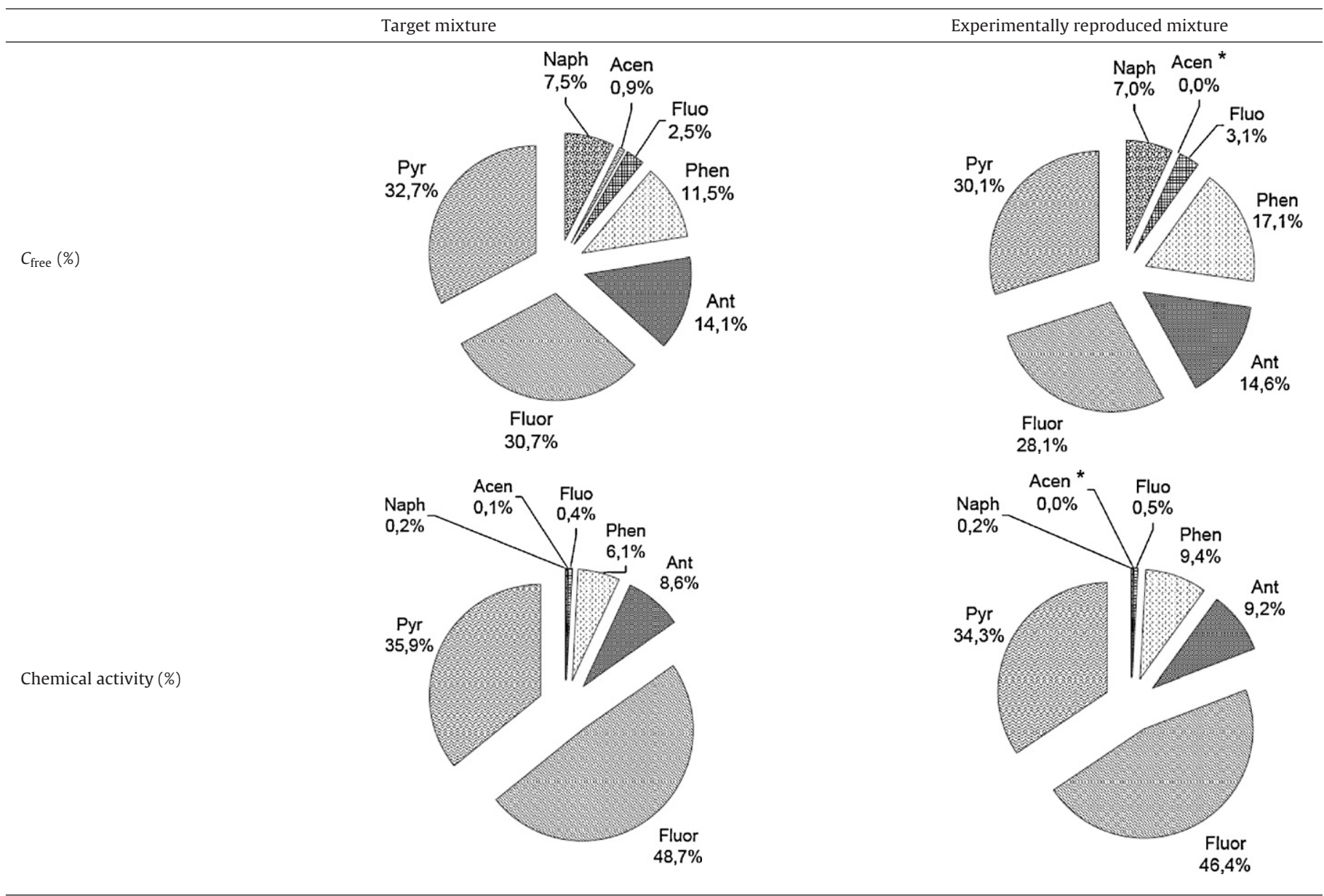

seawater mixture, but also on being able to control their levels. Based on the chemical activity-response curve determined above, the concentrations of the 7 PAHs in Bay of Algeciras sample were estimated to be non-toxic to $A$. franciscana. Therefore, in order to have an observable toxic response, passive dosing vials were prepared giving freely dissolved PAH exposure concentrations of $\times 1$, $\times 50, \times 100, \times 200$ and $\times 500$ the field sample concentrations. The ability of passive dosing to reproduce the PAH mixture of a seawater sample is shown in Fig. 3 showing the target concentrations and the experimentally reproduced concentrations by passive dosing for the $\times 500$ treatment as an example. The target (black bars) and reproduced (white bars) water concentrations were similar, as were the proportions of the different PAHs (see Fig. 3). Similar results were also obtained for $\times 200$ treatment, whereas water concentrations of several PAHs were below detection limits for the $\times 1, \times 50$ and $\times 100$ levels. In this figure the ratios between the target and measured concentrations are also shown, ranging between 0.98 and 1.16 . Phenanthrene was an exception, and had a slightly higher concentration by a factor of 1.58 with passive dosing.

Table 2 shows the mixture composition, giving the proportion of the individual PAHs on a $C_{\text {free }}$ and chemical activity basis for the target and passive dosing mixtures. The similarity between the two highlights the ability of passive dosing to faithfully reproduce mixture profiles. In fact, the maximum difference in chemical activities between target and measured proportions was 3\%, observed for phenanthrene. These results open up the possibility of using passive dosing for toxicity tests with complex mixtures of hydrophobic organics pollutants representing real world samples. CVs ranged between 0.003 and 0.012 highlighting the reproducibility. Note that the exposure concentrations were measured after the toxicity tests, confirming that the reservoir of test substances in PDMS

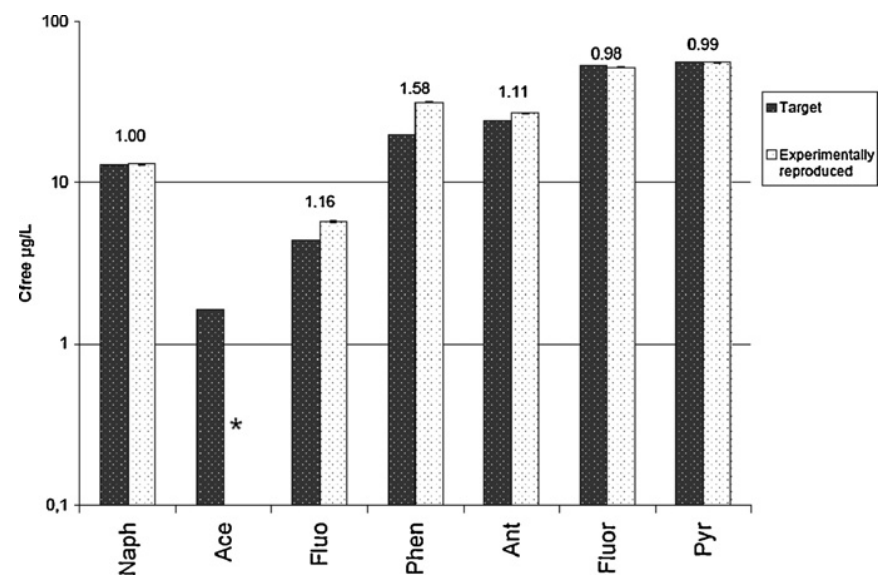

Fig. 3. Total concentration of each compound in sea water in the target mix ture $(\times 500)$ and in the actual mixture $(\times 500)$ obtained by passive dosing. PAHs were named as follows: naphthalene (Naph), acenaphthene (Ace), fluorene (Fluo), phenanthrene (Phen), anthracene (Ant), fluoranthene (Fluor) and pyrene (Pyr). Numbers above indicate the ratio between target and actual concentrations. *Indicates that the compound below detection limits. 
silicone was sufficiently large to compensate for losses without being significantly depleted.

\subsubsection{Toxicity tests with $A$. franciscana nauplii}

To determine whether there is toxicity due to the 7 PAHs in environmental samples from the Bay of Algeciras, and in the case of no toxicity to investigate how far the environmental concentrations are from a level that results in toxicity, toxicity test were performed with the same PAH profile but at different concentrations. The multiples tested were: $\times 1$ (concentrations of each PAH equal to those in the real sample), $\times 50, \times 100, \times 200$ and $\times 500(n=5)$. The test conditions were as above.

The toxicity results show that the concentration of PAHs naturally found in the seawater was not toxic to $A$. franciscana nauplii (Fig. 2). This was also the case for $\times 50$ concentration. However, with $\times 100, \times 200$ and $\times 500$ concentrations, the observed lethalities were $6 \%, 14 \%$ and $48 \%$, respectively. These toxicities were above those estimated from chemical activity response curve describing the toxicity of individual PAHs at their aqueous solubility. This is partly due to the fact that the mixtures had high concentrations of anthracene (see Fig. 2), which had a relatively higher toxicity to $A$. franciscana nauplii. The anthracene concentrations measured were $27.0 \mu \mathrm{g} \mathrm{L}^{-1}$ in $\times 500$ mixture, $11.9 \mu \mathrm{g} \mathrm{L}^{-1}$ in $\times 200$ mixture and $7.1 \mu \mathrm{g} \mathrm{L} \mathrm{L}^{-1}$ in $\times 100$ mixture.

These results might also be viewed as giving a safety factor for PAH toxicity in natural seawaters. In other words, concentrations would have to be increased by two orders of magnitude before the acute toxicity of the 7 PAHs starts to play a role in water of Bay of Algeciras. Of course, in the real world other compounds may contribute to, or even dominate, the toxicity at this site. Eventually toxicity assays based on passive dosing might be able to reproduce this full range of compounds.

\section{Conclusions}

This study shows that passive dosing has some important benefits for the testing of hydrophobic organic compounds (HOCs) in aquatic media. These include maintaining constant exposure concentrations and therefore giving higher reproducibility and a relative increase in toxicity. Passive dosing also allows quantification of the salting out effect, which is useful for aquatic toxicity assays with seawater. Finally, passive dosing can faithfully reproduce real mixtures of HOCs, such as PAHs, in toxicity tests, reproducing both the levels and proportions of the different compounds. This provides a useful approach for studying the toxicity of environmental mixtures of HOCs, with a view to investigating their toxicity but also for determining safety factors before such mixtures result in detrimental effects.

\section{Acknowledgements}

Elisa Rojo-Nieto thanks the Ministry of Education (Spain) for funding her research fellowship (FPU). The passive dosing work was funded by FP6 Integrated Project OSIRIS [COGE-037017]. Skilled technical help by Margit Fernqvist is gratefully acknowledged.

\section{Appendix A. Supplementary data}

Supplementary data associated with this article can be found, in the online version, at http://dx.doi.org/10.1016/ j.aquatox.2012.04.006.

\section{References}

Allred, P.M., Giesy, J.P., 1985. Solar radiation-induced toxicity of anthracene to Daphnia pulex. Environmental Toxicology and Chemistry 4 (2), 219-226.
Altenburger, R., Greco, W.R., 2009. Extrapolation concepts for dealing with multiple contamination in environmental risk assessment. Integrated Environmental Assessment and Management 5, 62-68.

Arias, A.H., Spetter, C.V., Freije, R.H., Marcovecchio, J.E., 2009. Polycyclic aromatic hydrocarbons in water, mussels (Brachidontes sp., Tagelus sp.) and fish (Odontesthes sp.) from Bahía Blanca Estuary, Argentina. Estuarine, Coastal and Shelf Science 85, 67-81.

Bandow, N., Altenburger, R., Lu $\square$ bcke-Von Varel, U., Paschke, A., Streck, G., Brack W., 2009. Partitioning-based dosing: an approach to include bioavailability in the effect-directed analysis of contaminated sediment samples. Environmental Science and Technology 43, 3891-3896.

Brown, R.S., Akhtar, P., Åkerman, J., Hampel, L., Kozin, I.S., Villerius, L.A., Klamer H.J.C., 2001. Partition controlled delivery of hydrophobic substances in toxicity tests using poly(dimethylsiloxane) (PDMS) films. Environmental Science and Technology 35, 4097-4102.

Code of Federal Regulation, 1982. Title 40, Appendix A to Part 423-126 Priority Pollutants. 47 FR 52304. United States of America.

Council Directive 2008/105/EC, 2008. On Environmental Quality Standards in the Field of Water Policy, Amending and Subsequently Repealing Council Directives 82/176/EEC, 83/513/EEC, 84/156/EEC, 84/491/EEC, 86/280/EEC And Amending Directive 2000/60/EC Of The European Parliament and of The Council. Official Journal of The European Union L348, 21/11/1996, P. 0084-0097.

de Maagd, P.G., ten Hulscher, D.T.E.M., van den Heuvel, H., Opperhuizen, A., Sijm, D.T.H.M., 1998. Physicochemical properties of polycyclic aromatic hydrocarbons: aqueous solubilities, $n$-octanol/water partition coefficients, and Henry's law constants. Environmental Toxicology and Chemistry 17 (2), 251-257.

Di Toro, D.M., McGrath, J.A., 2000. Technical basis for narcotic chemicals and polycyclic hydrocarbon criteria. II. Mixtures and sediments. Environmental Toxicology and Chemistry 19, 1971-1982.

ECETOC 1996. Monograph No. 26. Aquatic toxicity testing of sparingly soluble, volatile and unstable substances. European Centre for Ecotoxicology and Toxicology of Chemicals (ECETOC), Brussels.

Engraff, M., Solere, C., Smith, K.E.C., Mayer, P., Dahllöf, I., 2011. Aquatic toxicity of PAHs and PAH mixtures at saturation to benthic amphipods: linking toxic effects to chemical activity. Aquatic Toxicology 102, 142-149.

Gouliarmou, V., Smith, K.E.C., De Jonge, L.W., Mayer, P., 2012. Measuring binding and speciation of hydrophobic organic chemicals at controlled freely dissolved concentrations and without phase separation. Analytical Chemistry 84 (3), 1601-1608.

Hadjispyrou, S., Kungolos, A., Anagnostopoulos, A., 2001. Toxicity, bioaccumulation, and interactive effects of organotin, cadmium and chromium on Artemia Franciscana. Ecotoxicology and Environmental Safety 49, 179-186.

Huang, X.-D., McConkey, B.J., Babu, T.S., Greenberg, B.M., 1997. Mechanisms of photoinduced toxicity of photomodified anthracene to plants: inhibition of photosynthesis in the aquatic higher plant Lemna gibba (duckweed). Environmental Toxicology and Chemistry $16(8), 1707-1715$.

Hutchinson, T.H., Shillabeer, N., Winter, M.J., Pickford, D.B., 2006. Acute and chronic effects of carrier solvents in aquatic organisms: a critical review. Aquatic Toxicology $76(1), 69-92$.

Jonker, M.T.O., Muijs, B., 2010. Using solid phase micro extraction to determine salting-out (Setschenow) constants for hydrophobic organic chemicals. Chemosphere 80, 223-227.

Kiparissis, Y., Akhtar, P., Hodson, P.V., Brown, R.S., 2003. Partition-controlled delivery of toxicants: a novel in vivo approach for embryo toxicity testing. Environmenta Science and Technology 37, 2262-2266.

Koutsaftis, A., Aoyama, I., 2007. Toxicity of four antifouling biocides and their mixtures on the brine shrimp Artemia salina. Science of the Total Environment 387 , 166-174.

Lavens, P., Sorgeloos, P., 1996. Manual on the production and use of live food for aquaculture (FAO Fisheries technical paper No. 361). FAO, The Food and Agriculture Organization of the United Nations, Rome.

Law, R.J., Dawes, V.J., Woodhead, R.J., Matthiessen, P., 1997. Polycyclic aromatic hydrocarbons (PAH) in seawater around England and Wales. Marine Pollutution Bulletin 34, 306-322.

Mackay, D., Shiu, W.Y., Ma, K.-C., Lee, S.C., 2006. Polynuclear aromatic hydrocarbons (PAHs) and related aromatic hydrocarbons. In: Handbook of Physical-Chemica Properties and Environmental Fate for Organic Chemicals, 2nd edn. CRC Press, Boca Raton.

Mackay, D., Arnot, J.A., Petkova, E.P., Wallace, K.B., Call, D.J., Brooke, L.T., Veith, G.D. 2009. The physicochemical basis of QSARs for baseline toxicity. SAR and QSAR in Environmental Research 20,393-414

Mackay, D., Arnot, J.A., Wania, F., Bailey, R.E., 2011. Chemical activity as an integrating concept in environmental assessment and management of contaminants. Integrated Environmental Assessment and Management 7, 248-255.

Mallakin, A., McConkey, B.J., Miao, G.B., McKibbe, B., Snieckus, V., Dixon, D.G., Greenberg, B.M., 1999. Impacts of structural photomodification on the toxicity of environmental contaminants: anthracene photooxidation products. Ecotoxicology and Environmental Safety 43, 204-212.

Mathew, R., McGrath, J.A., DiToro, D.M., 2008. Modeling polycyclic aromatic hydrocarbon bioaccumulation and metabolism in time-variable early life-stage exposures. Environmental Toxicology and Chemistry 27 (7), 1515-1525

Mayer, P., Holmstrup, M., 2008. Passive dosing of soil invertebrates with polycyclic aromatic hydrocarbons: limited chemical activity explains toxicity cutoff. Environmental Science and Technology 42, 7516-7521. 
Mayer, P., Reichenberg, F., 2006. Can highly hydrophobic organic substances cause aquatic baseline toxicity and can they contribute to mixture toxicity? Environmental Toxicology and Chemistry 25, 2639-2644.

Mayer, P., Toräng, L., Glæsner, N., Jönsson, J.Å., 2009. Silicone membrane equilibrator: measuring chemical activity of nonpolar chemicals with poly(dimethylsiloxane) microtubes immersed directly in tissue and lipids. Analytical Chemistry 81, 1536-1542.

Mayer, P., Wernsing, J., Tolls, J., de Maagd, P.G.J., Sijm, D.T.H.M., 1999. Establishing and controlling dissolved concentrations of hydrophobic organics by partitioning from a solid phase. Environmental Science and Technology 33, 2284-2290.

Nunes, B.S., Carvalho, F.D., Guilhermino, L.M., Van Stappen, G., 2006. Use of the genus Artemia in ecotoxicity testing. Environmental Pollution 144, 453-462.

OECD, 2000. Guidance document on aquatic toxicity testing of difficult substances and mixtures; ENV/JM/MONO(2000)6. Organisation for Economic Co-operation.

Reichenberg, F., Mayer, P., 2006. Two complementary sides of bioavailability: accessibility and chemical activity of organic contaminants in sediments and soils. Environmental Toxicology and Chemistry 25, 1239-1245.

Reichenberg, F., Smedes, F., Jönsson, J.-Å., Mayer, P., 2008. Determining the chemical activity of hydrophobic organic compounds in soil using polymer coated vials. Chemistry Central Journal 2, 8.

Rewitz, K.F., Styrishave, B., Løbner-Olesen, A., Andersen, O., 2006. Marine invertebrate cytochrome $\mathrm{P} 450$ : emerging insights from vertebrate and insect analogies. Comparative Biochemistry and Physiology. C: Comparative Pharmacology 143 (4), 363-381.

Sarabia, R., Del Ramo, J., Varo, I., Diaz-Mayans, J., Torreblanca, A., 2002. Comparing the acute response to cadmium toxicity of nauplii from different populations of Artemia. Environmental Toxicology and Chemistry 21, 437-444.

Setschenow, J., 1889. Über Die Konstitution Der Salzlösungen Auf Grund Ihres Verhaltens Zu Kohlensäure. Zeitschrift für Physikalische Chemie 4, 117-128.
Smith, K.E.C., Dom, N., Blust, R., Mayer, P., 2010a. Controlling and maintaining exposure of hydrophobic organic compounds in aquatic toxicity tests by passive dosing. Aquatic Toxicology 98, 15-24.

Smith, K.E.C., Oostingh, G.J., Mayer, P., 2010b. Passive dosing for producing defined and constant exposure of hydrophobic organic compounds during in vitro toxicity tests. Chemical Research in Toxicology 23, 55-65.

Sorgeloos, P., Lavens, P., Léger, P., Tackaert, W., Versichele, D. 1986. Manual for the culture and use of brine shrimp Artemia in aquaculture. The Belgian Administration for Development Cooperation, The Food and Agriculture Organization of the United Nations, State University of Ghent, Belgium-Faculty of Agriculture $319 \mathrm{p}$.

Stevenson, R.W., 2003. Development and application of a model describing the bioaccumulation and metabolism of polycyclic aromatic hydrocarbons in a marine benthic food web. PhD Thesis. Simon Fraser University, Burnaby, BC Canada.

USEPA, 2002. Methods for Measuring the Acute Toxicity of Effluents and Receiving Waters to Freshwater and Marine Organisms. Fifth edition. EPA-821-R-02-012.

van Wezel, A.P., Opperhuizen, A., 1995. Narcosis due to environmental pollutants in aquatic organisms: residue-based toxicity, mechanism, and membrane burdens. Critical Reviews in Toxicology 25, 255-279.

Warner, A.H., Matheson, C., 1998. Release of proteases from larvae of the brine shrimp Artemia franciscana and their potential role during the molting process. Comparative Biochemistry and Physiology. B: Comparative Biochemistry 119 (2), 255-263.

Witt, G., 2002. Occurrence and transport of polycyclic aromatic hydrocarbons in the water bodies of the Baltic Sea. Marine Chemistry 79, 49-66. 